\section{Obésité et diabète}

\author{
La leptine, hormone \\ superstar de la satiété
}

La synthèse de la leptine, produit du gène ob sécrété par les adipocytes, est étroitement contrôlée par l'insuline, le régime alimentaire et d'autres hormones. Elle pourrait agir par l'intermédiaire de neuropeptides sur les centres hypothalamiques de la satiété, par exemple le neuropeptide $N P-Y$ et le glucagon-like peptide GLP-1. Son récepteur, de la famille de ceux des cytokines, vient d'être isolé et est fonctionnellement déficient chez les souris diabetic $(\mathrm{db} / \mathrm{db}) d u$ fait d'une mutation intronique activant un site d'épissage cryptique. Cependant, l'implication de ce système dans les obésités humaines reste incertaine. Les mécanismes génétiques gouvernant l'apparition de phénotypes de diabète de type II, marqués par la résistance à l'insuline et l'excès pondéral, pourraient être mieux compris grâce à l'étude d'un modèle récemment caractérisé chez le rat, qui développe spontanément cette symptomatologie.

\title{
Les facteurs régulateurs du gène ob
}

L'obésité est une affection multifactorielle, dont la forte composante génétique est maintenant bien démontrée chez l'homme [1]. A ce jour, on connaît au moins vingt loci répartis sur des chromosomes différents, qui présentent des polymorphismes associés à certains critères d'obésité. Cependant, aucune association suffisamment forte pour impliquer un gène particulier n'a encore pu être mise en évidence. Dans ce contexte, les modèles animaux d'obésité génétique peuvent fournir des indications précieuses sur la nature des gènes susceptibles de déterminer l'obésité chez l'homme. Trois gènes ont été clonés récemment grâce à la technique du clonage positionnel appliquée à des modèles murins d'obésité monogénique. Il s'agit des gènes agouti, fat et $o b$. Des informations concernant les gènes $d b$ et $t u b$ devraient apparaître dans la littérature dans un avenir proche.

Le clonage du gène $o b$ a révélé l'existence d'une nouvelle hormone, la leptine, sécrétée par la cellule adipeuse. C'est l'absence de leptine fonctionnelle qui détermine l'obésité de la souris $o b / o b$, modèle qui a permis le clonage du gène $\left(\mathrm{m} / \mathrm{s} n^{\circ} 12\right.$, vol. 10, p. 1337). Comme décrit récemment dans une mini-synthèse [2], le pouvoir amaigrissant de la leptine a été démontré par injection de la protéine recombinante. Bien que son mécanisme d'action reste encore mystérieux, on sait que la leptine provoque une réduction d'appétit et une augmentation de dépense énergétique. L'hypothèse qui prévaut actuellement, permettant d'expliquer ces effets, est celle d'une action au niveau de l'hypothalamus par l'intermédiaire de récepteurs, dont on pen- se qu'ils seraient codés par un autre gène de l'obésité, le gène $d b$. Le gène humain de la leptine présente un forte analogie avec le gène murin, et plusieurs études récentes ont révélé la présence de l'ARNm et de la protéine chez l'homme [3-6].

Le maintien d'un poids corporel constant chez l'individu normal suppose une régulation fine de la synthèse et de la sécrétion de leptine. En particulier, l'existence d'une boucle de régulation en retour est probable. Deux observations militent en faveur de cette hypothèse. Chez la souris $o b / o b$, l'une des deux mutations aboutissant à l'absence de protéine fonctionnelle introduit un codon stop dans la partie codante du gène. Chez ces animaux, l'ARNm muté est surexprimé, atteignant des niveaux d'expression vingt fois supérieurs à ceux des témoins. Cela suggère que la leptine intervient dans les mécanismes de régulation nécessaires à l'expression normale du gène [7]. La deuxième observation concerne les souris $d b / d b$, chez lesquelles un récepteur ou un effecteur de la leptine est muté, et qui synthétisent également en très grande quantité l'ARNm de $o b$ et la protéine [5, 8]. Ainsi, il semble qu'au moins un effecteur de la leptine soit également impliqué dans la régulation de l'expression du gène. Il est intéressant de noter qu'une synthèse exagérée de l'ARNm de $o b$ est également présente dans deux modèles d'obésité chez le rat: le rat Zucker $f a / f a$, porteur d'une mutation homologue à la mutation $d b$, et le rat «VMH», lésé au niveau de l'hypothalamus ventro-médian qui est le site potentiel d'expression du gène $d b / f a[8,9]$. Actuellement, on ne sait rien des protéines ou autres 
molécules relayant le signal leptine jusqu'à l'expression du gène dans la cellule adipeuse. Le clonage des gènes $d b$ ou $f a$ pourrait révéler l'un des composants de la boucle de régulation et ouvrir la voie à la découverte des effecteurs plus en aval.

L'expression du gène $o b$ dans la cellule adipeuse est également sous le contrôle de facteurs sanguins endogènes et exogènes. La première observation allant dans ce sens est la démonstration par notre groupe, que l'ARNm de $o b$ est induit dans le tissu adipeux de rats soumis à des injections répétées d'hydrocortisone ou de corticostéroïdes de synthèse (dexaméthasone et triamcinolone) à doses pharmacologiques [10]. L'induction du gène $o b$ est rapide et précède la réduction de prise alimentaire et de gain de poids chez les animaux traités. L'effet fortement stimulateur de la dexaméthasone sur l'ARNm de $o b$ peut être reproduit in vitro dans des adipocytes de rat en culture primaire, indiquant un effet direct des glucocorticoïdes sur l'expression du gène $o b[11]$. Ces résultats laissent penser que les effets de certains traitements pharmacologiques sur le poids corporel et l'appétit pourraient être dus à des modifications d'expression du gène $o b$.

D'autre part, des études récentes suggèrent que l'insuline pourrait être un modulateur de l'expression du gène $o b$. Cette hypothèse résulte de l'analyse de l'expression du gène dans des situations physiopathologiques où l'insulinémie est fortement modifiée. Parmi celles-ci, le cycle jeûne/réalimentation, naturel ou provoqué, étudié dans notre laboratoire [12]. Chez l'animal à jeun, l'ARNm de $o b$ n'est pratiquement plus détectable alors que son expression est restaurée à un niveau normal par la réalimentation. Les variations d'expression de l'ARNm de $o b$ sont parallèles aux variations d'insulinémie observées dans ces conditions. De plus, une seule injection d'insuline chez l'animal à jeun réinduit l'expression de l'ARNm au niveau de l'animal nourri. Enfin, des expériences d'injection simultanée d'insuline et de glucose ont démontré sans ambiguité que l'effet stimulateur de l'insuline sur l'expression de l'ARNm de $o b$ est indépendant des va- riations de glycémie. Cet effet de l'insuline est également détecté in vitro sur adipocytes de rat en culture primaire. Cependant, son amplitude est nettement plus faible, suggérant que l'environnement hormonal ou métabolique pourrait intervenir dans l'effet de l'insuline observé in vivo. Une autre étude réalisée dans le laboratoire de G. Ailhaud (Nice, France) montre également un effet stimulateur de l'insuline sur l'ARNm de $o b$ dans une lignée adipocytaire 3T3F442A [13]. De plus, les auteurs démontrent que la quantité de leptine sécrétée dans le milieu de culture par les adipocytes est aussi réglée par l'insuline. Enfin, dans la situation étudiée simultanément par l'équipe de D. Lane (Baltimore, USA) et celle de S. Brichard à Bruxelles (Belgique) le diabète insulinoprive provoqué par injection de streptozotocine, le signal $o b$ est considérablement diminué [14, 15]. Le traitement à l'insuline restaure un niveau normal d'expression [14]. L'ensemble de ces observations vont à l'appui de l'hypothèse que l'insuline serait un régulateur majeur de l'expression du gène $o b$ dans la cellule adipeuse.

D'autres situations physiologiques susceptibles d'influer sur l'expression du gène $o b$ ont été étudiées récemment, en particulier l'exposition au froid. Cependant, les résultats diffèrent suivant l'espèce. Le groupe de P. Trayhurn (Aberdeen, GB) rapporte qu'une exposition de 18 heures à $4^{\circ} \mathrm{C}$ provoque une forte diminution de l'ARNm de $o b$ chez la souris, cet effet étant totalement inversé par le retour au chaud. L'effet du froid est mimé par l'injection de noradrénaline, ce qui suggère l'intervention du système sympathique [16]. Cependant, chez le rat, le même type d'exposition au froid ainsi qu'une exposition chronique plus longue (3semaines) ne provoque pas de modification de l'expression de l'ARNm de $o b$ dans le tissu adipeux blanc [17]. Pour l'instant, il n'y a pas d'explication à cette différence. Physiologiquement, l'adaptation au froid s'accompagne d'une augmentation de prise alimentaire et de dépense énergétique, qui pourrait être gérée par l'organisme indépendamment d'un changement de concen- tration sanguine de leptine dans certaines espèces.

La régulation de l'expression du gène $o b$ chez l'homme n'a pas encore été abordée. Les données disponibles dans la littérature font état d'une augmentation de l'ARNm chez l'obèse, corrélée positivement avec l'index de masse corporelle [4]. Ces observations sont en accord avec l'hypothèse selon laquelle la leptine refléterait l'état des réserves de l'organisme. Cependant, elles impliquent que l'obèse humain est insensible, par un mécanisme qui reste à découvrir, aux effets anorexigène et stimulant de la dépense énergétique de la leptine. Certains obèses humains pourraient être porteurs de mutations sur des gènes codant pour un récepteur ou un effecteur en aval du gène $o b$, comme c'est le cas chez la souris $d b / d b$ et le rat $f a / f a$. A notre connaissance, aucune mutation du gène $o b$ entraînant une absence de leptine n'a été découverte chez l'homme, à ce jour.

La régulation physiopathologique de l'expression du gène $o b$ a été étudiée dans la plupart des cas par mesure des concentrations d'ARNm. Il sera important dans l'avenir de déterminer si les variations observées se traduisent par des variations de concentration sanguine de leptine. La disponibilité d'anticorps antileptine devrait permettre de répondre à cette question. Les mécanismes moléculaires qui régissent les modifications d'expression du gène et qui semblent intervenir en grande partie au niveau prétraductionnel, restent également à déterminer. La connaissance des facteurs régulateurs du gène $o b$ pourrait conduire à de nouvelles approches thérapeutiques du traitement de l'obésité

\section{Michèle Guerre-Millo}

Inserm U. 177, 15, rue de l'École de Médecine, 75270 Paris Cedex 06, France.

\section{Régis Saladin \\ Bart Staels \\ Johan Auwerx}

Inserm U.325, département d'athérosclérose, Institut Pasteur de Lille, 1, rue Calmette, 59019 Lille Cedex, France. 


\section{RÉFÉRENCES}

1. Ricquier D. Obésité et recherche en 1994. médecine/sciences $1994 ; 10$ : 1079-81. 2. Kahn A. Une confirmation : le produit du gène $o b$ est bien une hormone agissant comme un lipostat. médecine/sciences $1995 ; 11$ : 1463-4.

3. Masuzaki H, Ogawa Y, Isse N, Satoh N, Okazaki T, Shigemoto M, Mori K, Tamura N, Hosoda K, Yoshimasa Y, Jingami H, Kawada $T$, Nakao K. Human obese gene expression. Adipocyte-specific expression and regional differences in the adipose tissue. Diabetes 1995 ; 44 : 855-8.

4. Lonnqvist F, Arner P, Nordior L, Schalling

$\mathrm{M}$. Overexpression of the obese $(o b)$ gene in adipose tissue of human obese subjects. Nature Med 1995 ; 2 : 950-3.

5. Hamilton BS, Paglia D, Kwan AYM, Deitel

$M$. Increased obese mRNA expression in omental fat cells from massively obese humans. Nature Med 1995 ; 2 : 953-6.

6. Halaas JL, Gajiwala KS, Maffei M, CohenSL, Chait BT, Rabinowitz D,

Lallone RL, Burley SK, Friedman JM.

Weight-reducing effects of the plasma protein encoded by the obese gene. Science 1995 ; 269 : 543-6.

7. Zhang Y, Proenca R, Maffei M, Barone M, Leopold L, Friedman JM. Positional cloning of the mouse obese gene and its human homologue. Nature 1995 ; 372 : 425-32.

8. Maffei M, Fei H, Lee GH, Dani C, Leroy

$\mathrm{P}$, Zhang Y, Proenca R, Négrel R, Ailhaud

G, Friedman JM. Increased expression

in adipocytes of $o b$ mRNA in mice with lesions

of the hypothalamus and with mutations

at the $d b$ locus. Proc Natl Acad Sci USA

1995 ; 92 : 6957-60.

9. Ogawa Y, Masuzaki H, Isse N, Okazaki T, Mori K, Shigemoto M, Satoh N, Tamura N,

Hosoda K, Yoshimasa Y, Jingami H,

Kawada T, Nakao K. Molecular cloning of

rat obese cDNA and augmented gene expression

in genetically obese Zucker fatty ( $\mathrm{fa} / \mathrm{fa}$ )

rats. J Clin Invest 1995 ; 96 : 1647-52.

10. De Vos P, Saladin R, Auwerx J, Staels B.

Induction of $o b$ gene expression by corticosteroids is accompagnied by body weight loss and reduced food intake. J Biol Chem 1995 ; 270 : 15958-61.

11. Murakami T, Iida M, Shima K. Dexamethasone regulates obese expression in isolated rat adipocytes. Biochem Biophys Res Commun 1995 ; 214 : 1260-7.

12. Saladin R, De Vos P, Guerre-Millo M, Leturque A, Girard J, Staels B, Auwerx J. Transient increase in obese gene expression after food intake or insulin administration. Nature 1995 ; 377 : 527-9.

13. Leroy P, Dessolin S, Villageois $P$, MoonBC, Friedman JM, Ailhaud G, Dani C. Expression of $o b$ gene in adipose cells : Regulation by insulin. J Biol Chem 1996 ; 271

(sous presse).

14. Macdougald OA, Hwang CS, Fan H,

Lane DM. Regulated expression of the obese gene product (leptin) in white adipose tissue and 3T3-L1 adipocytes. Proc Natl Acad Sci USA 1995 ; 92 : 9034-7.

15. Becker DJ, Ongemba LN, Brichard V,
Henquin JC, Brichard SM. Diet-and diabetesinduced changes of ob gene expression in rat adipose tissue. FEBS Lett 1995 ; 371 : 324-8.

16. Trayhurn P, Ducan JS, Rayner DV. Acute cold-induced suppression of $o b$ (obese) gene expression in white adipose tissue of mice : mediation by the sympathetic system. Biochem J 1995 ; 311 : 729-33.

17. Moinat M, Deng C, Muzzin P, AssimacopoulosJeannet F, Seydoux J, Dulloo AG,

Giacobino JP. Modulation of obese gene expression in rat brown and white adipose tissues.

FEBS Lett 1995 ; 373 : 131-4. 